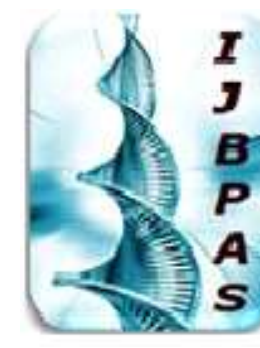

International Journal of Biology, Pharmacy and Allied Seiences (IJBPAS)

'A Bridge Batusen Caboratory and Q Q and

WwW.ijbpas.com

GASTRORETENTIVE FLOATING MATRIX TABLET OF METFORMIN HYDROCHLORIDE

\title{
KAMERE $\mathbf{K}^{1^{*}}$, GOPALAKRISHNA $\mathrm{SV}^{2}$, SUBBAREDDY GV $^{3}$
}

1: Research Scholar, JNTUA, Anantapuramu, Andhra Pradesh, India

2: Vagdevi college of Pharmacy \& Research centre, Brahmadevam (V), Muthukur (M), Nellore, Dt.-524 346, Andhra Pradesh, India

3: JNTUA College of Engineering, Pulivendula, YSR Kadapa Dt.-516390, Andhra Pradesh, India

*Corresponding Author: Kamere K: E Mail: pharmarxpro@gmail.com

Received 16 ${ }^{\text {th }}$ Dec. 2019; Revised $3^{\text {rd }}$ Jan. 2020; Accepted $15^{\text {th }}$ Feb 2020; Available online $1^{\text {st }}$ Nov. 2020 https://doi.org/10.31032/IJBPAS/2020/9.11.5241

\begin{abstract}
It is always challenging to prepare the floating gastro retentive formulation of the high dose drug. The present research was aimed to prepare gastro retentive controlled release, matrix tablets of metformin, using the combination of different ionic, anionic and polyanionic polymers with HPMC. Formulations were prepared using sodium alginate, pullulan, kappa carrageenan, xanthan gum, poloxamer 68 in combination with HPMC K15M. All matrix tablets were prepared by direct compression method and evaluated for swelling, floating adhesive period and drug release. All the tablets showed acceptable physicochemical properties. Statistical analyses of data revealed that tablets prepared using HPMC K15M and kappa carrageenan, formulation $\mathrm{F} 2$, is best in terms of showing excellent floating properties, extended adhesion periods and sustained drug release characteristics with similarity factor as 92 on comparison with the theoretical release of the drug. The combination of HPMC K15M and kappa carrageenan can be further optimized by applying the appropriate statistical design.
\end{abstract}

Keywords: Metformin, Gastro retentive, HPMC K15M, Kappa carrageenan 


\section{INTRODUCTION}

Metformin Hydrochloride is an oral hypoglycemic agent, which belongs to the class of biguanide derivatives [1]. Metformin has an absolute bioavailability of 50-60\%, when administered orally due to its incomplete absorption, as it doesn't remain in the stomach for a longer period of time [2]. It has a biological half-life 1.5-1.6 $\mathrm{h}$, and the main site of absorption for metformin is proximal small intestine of the GIT [3, 4]. Many approaches have been reported for increasing the bioavailability of metformin by preparing controlled release and gastro retentive dosage forms $[\mathbf{5}, \mathbf{6}]$.

The exhaustive literature research elucidates that several approaches have been tried for the preparation of gastro retentive metformin formulations. Stepensky et al., 2001, established PK-PD rationale for the development of metformin CR formulations and it was concluded that GRDDS of this drug can be clinically advantageous [7]. Colo et al., prepared $\mathrm{pH}$-controlled peroral delivery of metformin and it was found that this mode of administration might allow the use of lower therapeutic doses of the drug compared to existing immediate or sustainedrelease products, thus minimizing side effects [8]. Ali et al., (2007) prepared Hydrodynamically balanced system for metformin as a single unit floating capsule, Gamma Scintigraphic studies revealed that the optimized HBS capsule was retained in the gastric region (stomach) for a prolonged period and pharmacokinetic studies showed an increase in AUC as compared to immediate release capsules of metformin [9]. Tack-Oon Oh. et al. (2013) prepared, highly Porous Gastro retentive metformin Tablets using a Sublimation method with $24 \mathrm{~h}$ floating time. Pharmacokinetic studies revealed that the mean plasma concentration of the GR tablets after oral administration was greater than the concentration of Glucophage XR [10]. Anita K. Lalloo, et al. (2012) developed a controlled release formulation of metformin using swelling mechanism and compared it with gastro retentive marketed formulation, Glumetza ${ }^{\circledR}$, of the same drug for understanding the relationship between the drug release rate, absorption rate and position in the gastrointestinal tract for GR and $\mathrm{CR}$ formulations. Diet was identified as the critical determinant of gastro retention in this research, as also reported in the clinic for Glumetza ${ }^{\circledR}$ and Proquin XRTM. Optimal gastric retention can hence be achieved by modulating the size/swelling rate of the 
dosage form together with the erosion rate [11].

An important factor for the development of gastro retentive dosage form is the selection of suitable hydrophilic polymer, which provides acceptable flotation characteristics and release of the drug substance. Drug dissolution from hydrophilic matrix systems is related to the entry of water into the matrices. Li et al., (2008) suggested that many physicochemical phenomena occur simultaneously during dissolution [12]. The release mechanism of the drug from the polymeric matrix has been explained by many researchers, but in most of the studies, hydroxy propyl methyl cellulose (HPMC) is used as polymeric floating matrix system [13-15]. But the combination of HPMC with other ionic and anionic polymeric substances and their effect on the release of the drug has not been explored much. Dorozynski et al. (2011) used carrageenans and their mixtures with HPMC for preparing gastro retentive drug delivery systems of 1-dopa. The formulations showed linear increase in the releasing rate constantly. In such formulations, carrageenans can modify the properties of polymeric matrices, to obtain tailor-made materials for drug delivery systems [16]. In another attempt, the effect of different viscosity grade HPMC polymers was checked on the Gastro retentive dosage form of metformin $\mathrm{HCl}$ [17].

This research has been conducted to check the combined effect of HPMC and other polymers on the release and gastro retentive properties of the formulation. The dose of metformin for a gastro retentive $\mathrm{SR}$ formulation was taken as $500 \mathrm{mg}$ and was calculated in the same way as done by Mandal et al., using the equation given by Rawlins with the available pharmacokinetic data given by Defang et al. (2005) [18-20]. The objective of the present research work was to prepare, characterize and evaluate floating, gastro retentive drug delivery systems of metformin using a combination of polymeric matrix, in order to achieve an extended retention of the drug in the upper GIT, which may result in enhanced absorption, improved bioavailability, decreased dosing frequency and increased patient compliance.

\section{MATERIALS AND METHODS}

\section{Materials}

Metformin was obtained as a gift sample from Sanofi-Aventis Ltd., Ankleshwar. Sodium bicarbonate, sodium alginate and xanthan gum were procured from Sulab Reagents, Suvidhinath laboratories,

Baroda, HPMC K15M and carbopol 934 were procured from Astron Chemicals, 
Ahmedabad, poloxamer 68 Signet was purchased from Chemical Co. Pvt. Ltd., Mumbai, kappa-carrageenan was procured from Rajesh Chemicals, Vadodara. All other excipients were procured from the local market.

\section{Preliminary studies}

From the literature review, it was found that HPMC is a good release retarding polymer and gives $\mathrm{pH}$-dependent drug release. Hence, for weakly basic drugs, it gives increased release in acidic $\mathrm{pH}$. As the dose of the drug is more so $\mathrm{K} 15 \mathrm{M}$ grade was selected as release retarding polymer. For optimizing the quantity of HPMC K15M various batches of floating matrix tablet of metformin were prepared.

The amount of HPMC K15M was varied from $10 \%$ to $30 \%$ and the quantities of other additives were fixed (metformin 50\%, sodium bicarbonate $\left(\mathrm{NaHCO}_{3}\right) \quad 18 \%$, Magnesium stearate 1\%). The weight of Microcrystalline cellulose was adjusted to keep the total weight of the tablet as 1000 mg. Prepared formulations were evaluated for the floating lag time and total floating time.

\section{Formulation of metformin floating tablets}

Tablets containing $500 \mathrm{mg}$ of metformin were prepared, according to the design depicted in Table 1, by direct compression technique. The respective powders, namely drug, release-retarding polymer(s) (HPMC $\mathrm{K} 15 \mathrm{M}$ and sodium alginate/kappacarrageenan/pullulan/xanthangum/poloxamer 68/carbopol 934 P), a gas-forming agent, NaHCO3, were passed through sieve no. 20, separately. Mixing of powders was carried out using a pestle and mortar for $10 \mathrm{~min}$. microcrystalline cellulose and magnesium stearate were then added to the mixed powders. Mixing was continued for another 5 min. Finally, $1000 \mathrm{mg}$ of each mixture were weighed and fed manually into the die of a rotary tablet compression machine (Cronimach Instrument, Ahmedabad, India), equipped with capsule shaped punch die set, to produce the desired tablets. The hardness of the tablets was adjusted at $5 \mathrm{~kg} / \mathrm{cm} 2$ using a monsanto hardness tester (M. Shah and company, Vadodara, India).

\section{Evaluation of prepared formulations}

\section{(Table 2)}

\section{Tablet weight variation}

Twenty tablets were randomly selected and accurately weighed. Results are expressed as mean values $\hat{A} \pm S D$.

\section{Drug content uniformity}

Ten tablets were individually weighed and crushed. A quantity of powder equivalent to the mass of one tablet $1000 \mathrm{mg}$ was extracted in $100 \mathrm{ml}$ of $0.1 \mathrm{~N} \mathrm{HCl}$. The solution was 
filtered through a cellulose acetate membrane (0.45 Â $\mu \mathrm{m})$. The drug content was determined by UV spectroscopy (Shimadzu UV 1800 Double beam spectrometer, Shimadzu Corporation, Japan) at a wavelength of $230 \mathrm{~nm}$ after a suitable dilution with $0.1 \mathrm{~N} \mathrm{HCl}$.

\section{Tablet friability}

According to the IP specifications [21], 10 tablets were randomly selected from each batch and placed in the drum of a tablet friability test apparatus (DBK instruments, Electro quip Inst., Ahmedabad). The drum was adjusted to rotate 100 times in $4 \mathrm{~min}$.
The swelling behavior of the tablets was determined, in triplicate, according to the method described by Dorozynski et al. [22]. Briefly, a tablet was weighed (W1) and placed in in the petridish with $20 \mathrm{ml}$ of $\mathrm{HCl}$ $(0.1 \mathrm{~N})$, maintained at $37 \hat{\mathrm{A}} \pm 0.5 \hat{\mathrm{A}}^{\circ} \mathrm{C}$. After 8 $\mathrm{h}$ the tablets were removed from the petridish and the swollen tablet was then reweighed (W2) [23]. The swelling index (SI) was calculated using following formula.

$$
\text { Swelling Index }=\frac{(W 2-W 1)}{W 1}
$$

Where, W2 is the weight of the swollen tablets, and W1 is the initial weight of the tablets. The size of tablets, before and after swelling, was also measured

\section{Tablet swelling ability}

Table 1: The composition (in percentage) of metformin HCl floating matrix tablets

\begin{tabular}{|c|c|c|c|c|c|c|c|}
\hline S. No. & Ingredients & F1 & F2 & F3 & F4 & F5 & F6 \\
\hline 1 & MetforminHcl & 50 & 50 & 50 & 50 & 50 & 50 \\
\hline 2 & HРMC К15M & 17 & 17 & 17 & 17 & 17 & 17 \\
\hline 3 & Sodium bicarbonate & 18 & 18 & 18 & 18 & 18 & 18 \\
\hline 4 & Sodium Alginate & 8 & - & - & - & - & - \\
\hline 5 & $\square$-Carrageenan & - & 8 & - & - & - & - \\
\hline 6 & Pullulan & - & - & 8 & - & - & - \\
\hline 7 & Xanthan gum & - & - & - & 8 & - & - \\
\hline 8 & Poloxamer 68 & - & - & - & - & 8 & - \\
\hline 9 & MCC & 6 & 6 & 6 & 6 & 6 & 14 \\
\hline 10 & Mg stearate & 1 & 1 & 1 & 1 & 1 & 1 \\
\hline
\end{tabular}

Table 2: Physical evaluation of prepared formulations

\begin{tabular}{|c|c|c|c|c|}
\hline Batch code & Weight uniformity & Hardness* $\left(\mathrm{kg} / \mathrm{cm}^{2}\right)$ & Drug content* $(\%)$ & Friability* (\%) \\
\hline F1 & Complies & $5.7 \pm 0.95$ & $100.74 \pm 0.94$ & $0.25 \pm 0.09$ \\
\hline F2 & Complies & $4.2 \pm 0.62$ & $98.56 \pm 1.25$ & $0.23 \pm 0.12$ \\
\hline F3 & Complies & $4.0 \pm 0.28$ & $98.73 \pm 1.37$ & $0.13 \pm 0.10$ \\
\hline F4 & Complies & $4.7 \pm 0.54$ & $99.46 \pm 0.59$ & $0.29 \pm 0.20$ \\
\hline F5 & Complies & $4.6 \pm 0.65$ & $100.98 \pm 0.94$ & $0.44 \pm 0.16$ \\
\hline F6 & Complies & $5.1 \pm 0.98$ & $99.57 \pm 0.99$ & $0.13 \pm 0.11$ \\
\hline
\end{tabular}

\section{In vitro buoyancy studies}

The floating behavior of the tablets was visually determined, in triplicate, according to the floating lag time method described by
Rosa et al. [24]. Briefly, a tablet was placed in a glass beaker, containing $200 \mathrm{ml}$ of $0.1 \mathrm{~N}$ $\mathrm{HCl}$, maintained in a water bath at $37 \pm 0.5$ ${ }^{\circ} \mathrm{C}$. The floating lag time, " the time between 
tablet was placed in a glass beaker with $\mathrm{HCl}$ and its buoyancy" and total floating duration, "the time during which tablet remains buoyant", were recorded.

\section{Tablet adhesion retention period}

The adhesion retention period of the tablets was evaluated, in triplicate, by an in vitro method reported by Nakamura et al. (1996) for measuring the nasal mucoadhesion of some water-soluble polymers [25]. Briefly, an agar plate $(2 \%, \mathrm{w} / \mathrm{w})$ was prepared in 0.1 $\mathrm{N} \mathrm{HCl}(\mathrm{pH}$ 1.2). A side of the tablet was wetted with $0.1 \mathrm{~N} \mathrm{HCl}$ and attached to the center of agar plate by applying a light force with a fingertip [26]. Five minutes later, the agar plate was attached to a USP disintegration test apparatus and moved up and down in $0.1 \mathrm{~N} \mathrm{HCl}(\mathrm{pH} 1.2)$ at $37 \pm 0.5^{\circ} \mathrm{C}$ (Figure 1). The adhering tablet on the plate was immersed into the solution at the lowest point and got out of the solution at the highest point. The retention period of the tablet on the plate was noted visually.

\section{Drug release studies}

Drug release study of the prepared floating tablets was performed, in triplicate, in a USP Dissolution Tester Apparatus, type-II (Paddle method) (Electrolab TDT-08L, Purvi enterprise, Gujarat, India) at $37 \pm 0.5{ }^{\circ} \mathrm{C}$. The paddles rotated at a speed of $100 \mathrm{rpm}$. The tablets were placed into $900 \mathrm{ml}$ of $0.1 \mathrm{~N} \mathrm{HCl}$ solution ( $\mathrm{pH}$ 1.2). Aliquots of $5 \mathrm{ml}$ were withdrawn from the dissolution apparatus at different time intervals and filtered through a cellulose acetate membrane $(0.45 \mu \mathrm{m})$. The drug content was determined spectrophotometrically at a wavelength of $230 \mathrm{~nm}$, as mentioned before. At each time of withdrawal, $5 \mathrm{ml}$ of fresh medium was replaced into the dissolution flask, to maintain the sink condition. The release of the prepared gastro retentive formulations was compared with the theoretical release of the drug by calculating similarity and dissimilarity factor $[\mathbf{2 7}, \mathbf{2 8}]$.

Similarity factor means the comparison of resemblance in the release pattern of two comparative formulations. Generally, a similarity factor in the range of $50-100$ is acceptable according to the US FDA. It can be calculated using the following equation:

$$
\mathrm{f} 2=50 . \log \left\{\left[1+\frac{1}{n} \sum_{t=1}^{n} R t-T t^{2}\right]^{-0.5} \times 100\right\}
$$

Where, $\mathbf{n}$ is the number of dissolution sample times, $\mathbf{R t}$ and $\mathrm{Tt}$ are the individual or the mean percent dissolved at each time point, $t$, for the reference and test dissolution profiles, respectively.

The similarity factor should be between 0 and 100. It is 100 when two comparative groups of reference and test are identical and approaches 0 as the dissimilarity increases.

Dissimilarity factor calculates the difference in percent dissolved between reference and 
test at various time intervals. It can be calculated using the following equation:

$$
\mathbf{f 1}=\{[\mathbf{S} t=\mathbf{1 n}|\mathbf{R t}-\mathbf{T t}|] /[\mathrm{S} t=1 \mathrm{nRt}]\} \times \mathbf{x 1 0 0}
$$

\section{Drug excipient compatibility study}

There is always the possibility of drugpolymer interaction in any formulation. The FTIR of pure drug and physical mixture of drug metformin Hydrochloride and polymers HPMC K15M and kappa carrageenan was performed using Fourier Transform Infrared Spectrophotometer (8400S DRS, Shimadzu Corp). The amount of drug and polymer of the physical mixture was same as that in the optimized batch. The pure drug and physical mixture were then separately mixed with IR grade $\mathrm{KBr}$. This mixture was then scanned over a wave number range of 4000 to 400 cm-1.

\section{RESULTS AND DISCUSSION}

\section{Preliminary studies}

For optimizing the quantity of release retarding polymer, various batches of floating matrix tablet of metformin Formulations was prepared, using HPMC $\mathrm{K} 15 \mathrm{M}$ by fixing the quantities of other additives. Results indicated that batches formulated with low polymer concentration got disintegrated in $0.1 \mathrm{~N} \mathrm{HCl}$ and the tablets prepared with the highest polymer concentration could not float.
It was found that HPMC K15M was giving satisfactory results from the concentration ranging $15-25 \%$. The tablets prepared with $15 \%$ of HPMC could float for $3 \mathrm{~h}$ with $10 \mathrm{sec}$ lag time. It was concluded that as the concentration of HPMC was increased, the floating lag time as well as floating time was also increasing. It was decided to take the minimum amount of HPMC $\mathrm{K} 15 \mathrm{M}$ giving the satisfactory results, for further study to check the effect of the combination of other polymers with HPMC on gastro retention of tablets.

\section{Physical properties of floating tablet}

The physical properties of the floating tablets were found to be satisfactory for all the batches. The hardness of all the batches was found to be in the range of $4-5.7 \mathrm{~kg} / \mathrm{cm} 2$. Drug content of all the formulations was near $100 \%$ and friability was found to be within limits given in official books.

$*_{n}=3$, average of three determinations \pm SD

\section{In vitro buoyancy studies}

All the prepared formulations showed the floating lag time of fewer than 30 seconds, and tablets could float for more than $8 \mathrm{~h}$, as shown in Table 3. This indicates that a combination of polymers had no effect on lag time and total floating time of the prepared formulations, in this particular ratio. 


\section{Tablet swelling ability}

The swelling index of formulation 2, with kappa carrageenan, was found to be highest Till $8 \mathrm{~h}$, this formulation was intact and was deformed after $24 \mathrm{~h}$, showing that the formulation would eventually go out of the stomach after the release of the drug, which is desirable. The minimum swelling index was calculated for the formulation with sodium alginate which means that the polymer doesnâ€ $€^{\mathrm{TM}_{\mathrm{t}}}$ promote water uptake by polymeric matrices containing HPMC. This formulation was intact even after $24 \mathrm{~h}$, which suggests that formulation is not getting eroded eventually.

\section{Tablet adhesion retention period}

The formulations gave the tablet retention between the range of 18 to $94 \mathrm{~min}$. Tablets prepared with the HPMC K15M and kappacarrageenan gave maximum adhesion retention of $93.50 \mathrm{~min}$ and formulation prepared using sodium alginate and HPMC K15M exhibited minimum adhesion retention period.

\section{Drug release studies}

From this study, it was found that formulation F2 (formulation with HPMC $\mathrm{K} 15 \mathrm{M}$ and kappa-carrageenan) was giving almost same release pattern as that of a theoretical release pattern of the drug. Formulation F4 and F5 with xanthan gum and poloxamer 68, respectively, could not sustain the release of the drug for more than $6 \mathrm{~h}$. After 2 h, $50 \%$ of the drug was released from these formulations. Hence, these polymers arenot the good release-retarding polymers for preparing matrix formulations. Formulations F1, F3 and F6 with sodium alginate, pullulan and only HPMC, respectively, showed the delayed release of the drug from the matrix. Only $85 \%$ of the drug was released from these formulations in $8 \mathrm{~h}$. All the formulations can be optimized by varying the concentration of the release retarding polymer (Figure 2).

The similarity and dissimilarity factor of the release data was calculated and formulation F2, with kappa carrageenan, gave $\mathrm{f} 2$ value as 92 and $\mathrm{f} 1$ value as 1. Formulation F3 and F4 doesn't pass the test as the values were out of the range. Other formulations i. e F1, F5 and F6 had the $\mathrm{f} 2$ value like 58, 53, 53 respectively.

\section{Drug excipient compatibility study}

The FTIR scan of the drug, polymers and physical mixture of drug and polymer was taken and the distinct peaks of metformin were present in the physical mixture of metformin and polymer, which proves that there was no interaction between drug and polymer. 
Table 3: Results table for buoyancy, swelling ability and tablet retention period

\begin{tabular}{|c|c|c|c|c|c|c|}
\hline \multirow[t]{2}{*}{ Formulation } & \multirow[t]{2}{*}{$\begin{array}{c}\text { Lag } \\
\text { Time*}^{*}(s)\end{array}$} & \multirow[t]{2}{*}{$\begin{array}{l}\text { Floating } \\
\text { Time* }^{*}(\mathrm{~h})\end{array}$} & \multirow{2}{*}{$\begin{array}{l}\text { Tablet adhesion } \\
\text { retention period* } \\
\text { (min.) }\end{array}$} & \multirow[t]{2}{*}{$\begin{array}{l}\text { Swelling index } \\
\text { (ratio) }\end{array}$} & \multicolumn{2}{|c|}{$\begin{array}{c}\text { Physical appearance of the tablet } \\
\text { After swelling }\end{array}$} \\
\hline & & & & & 8h (width) & $24 \mathrm{~h}$ \\
\hline F1 & $15.25 \pm 1.20$ & $>8$ & $18.25 \pm 2.41$ & 1.734 & Intact $2 \mathrm{~cm}$ & intact \\
\hline F2 & $10.71 \pm 2.36$ & $>8$ & $93.50 \pm 3.36$ & 3.864 & $2.2 \mathrm{~cm}$ & deformed \\
\hline $\mathbf{F 3}$ & $30.50 \pm 3.17$ & $>8$ & $66.41 \pm 3.42$ & 2.755 & $1.8 \mathrm{~cm}$ & deformed \\
\hline F4 & $12.07 \pm 1.70$ & $>8$ & $42.12 \pm 4.25$ & 2.851 & deformed & deformed \\
\hline F5 & $15.65 \pm 2.20$ & $>8$ & $17.10 \pm 2.45$ & 2.501 & deformed & deformed \\
\hline F6 & $30.75 \pm 4.96$ & $>8$ & $21.41 \pm 2.15$ & 2.827 & $1.7 \mathrm{~cm}$ & deformed \\
\hline
\end{tabular}

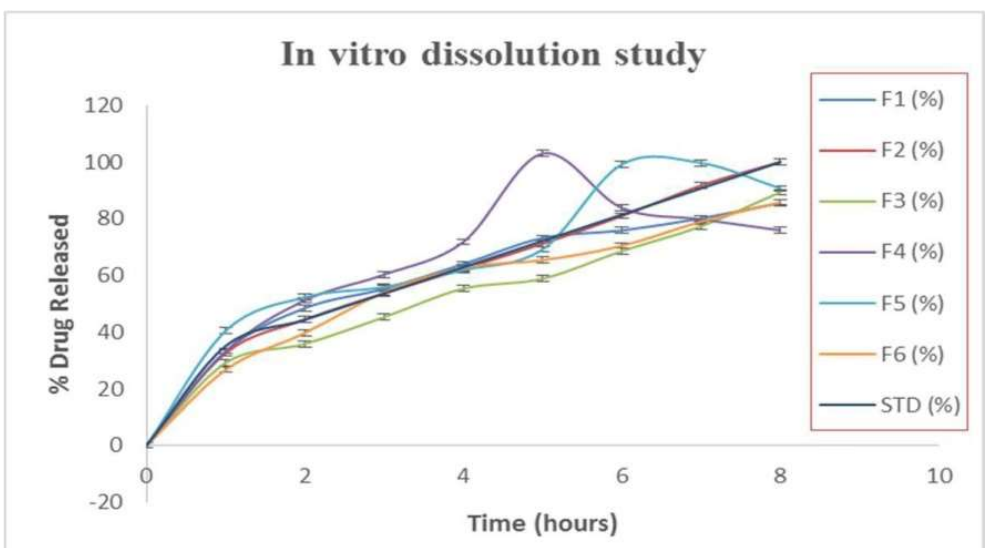

Figure 1: It shows in vitro dissolution profiles of different floating tablet formulations in $0.1 \mathrm{~N} \mathrm{HCl*}$, *n=3, average of three determinations \pm SD
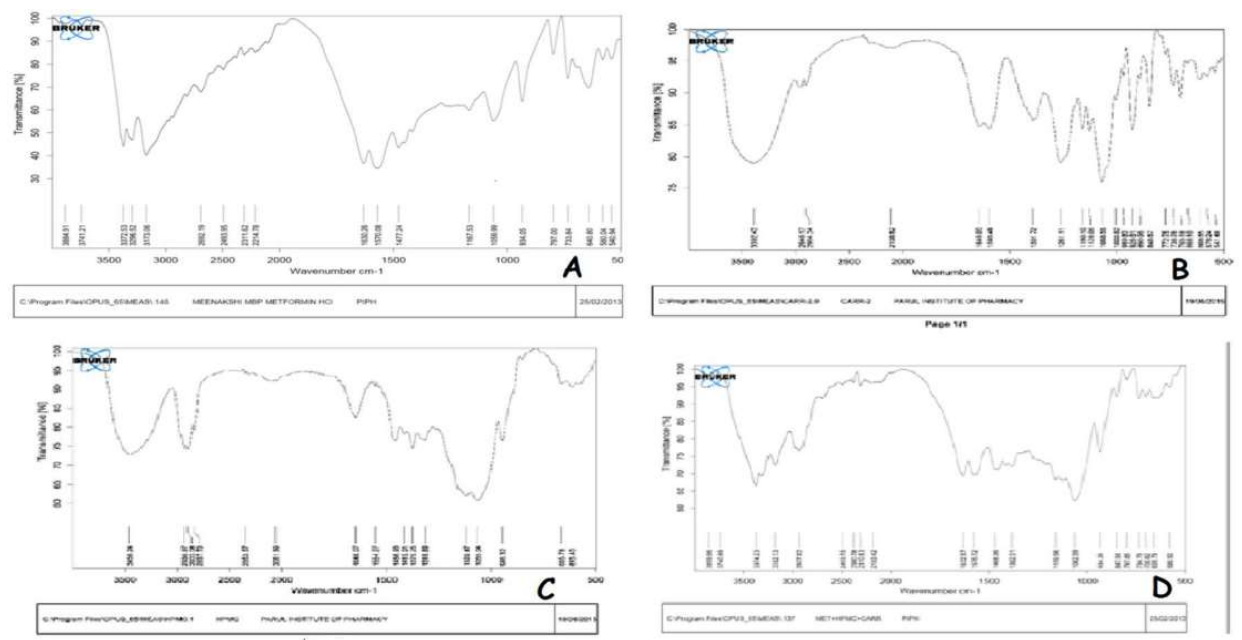

Figure 2: FTIR scan obtained for metformin hydrochloride (A), kappa carrageenan (B), HPMC K15M (C) and optimized formulation (D) 


\section{DISCUSSION}

The results of physical evaluation of the prepared dosage forms gave acceptable physical characteristics. The assay for drug content indicated acceptable content uniformity in the prepared tablets. Previous literature reported that viscosity of the gelforming polymer influences the in vitro buoyancy [29]. All the formulations had floating lag time less than 31 seconds and floating time, more than $8 \mathrm{~h}$, which means that variation of the polymers along with HPMC had no effect on the floating properties of the tablets. This shows that the flotation is dependent on the amount of sodium bicarbonate present in the formulation. It has been reported earlier that strength of gel layer changes with the increase in polymer proportion which in turn will affect flotation of the tablet [30]. Hence, as the amount of sodium bicarbonate and HPMC was same for all the formulations, the floating properties were also similar.

The swelling index of formulation 2, with kappa carrageenan was found to be higher which proves the findings of Dorozynski et al., (2011) where he showed that application of mixtures of carrageenan and HPMC, increase the swelling capacity of HBS formulations and suggested that this combination can be directly utilized as a starting

point in the development of various controlled release formulations [16]. The minimum swelling index was calculated for the formulation with sodium alginate which means that the polymer doesn't promote water uptake by polymeric matrices containing HPMC. This can be attributed to the $\mathrm{pH}$-dependent solubility of sodium alginate as suggested by Timmins et al., (1997), where it was proved that sodium alginate hydrates and swells in alkaline $\mathrm{pH}$ and doesn't form the gel layer in the stomach [31].

Agar plates were used to check the comparative adhesion retention of the prepared formulations as it is negative charges, same as that of mucin covering the mucous membrane [25]. Agar gel contains large numbers of negatively charged carboxyl and sulfate groups; therefore, they have a high negative charge. On performing the comparative adhesion retention period study for prepared tablets, it was found that formulation with kappa-carrageenan was retained on the agar plate for a longer period of time as compared to other formulations. As proved by Campo et al., (2009), carrageenan is a high molecular weight sulfated polysaccharides and its high 
adhesion period may be due to hydrogen bonding or ionic interaction with agar [32]. Formulations prepared with poloxamer 68 and sodium alginate showed a minimum retention period as they have lower ability to interact with agar.

Tatavarti et al., (2004), proved that incorporation of anionic polymers, in HPMC matrices is useful for developing a $\mathrm{pH}$ independent release profile for weakly basic drugs [33]. The present study also revealed that incorporation of kappa-Carrageenan, a polyanionic polymer, in an HPMC matrix of metformin showed the best release pattern. This combination in F2 formulation showed an almost similar release pattern as that of a theoretical release pattern of the drug with maximum f2 value. Singh et al., (2011) presented the release behavior of drugs from different natural polymers and gums [34]. They found that the presence of xanthan gum in the formulation can retard the release of the drug. Whereas, during the present study, formulation F4 prepared with xanthan gum could not sustain the release of the drug for more than $6 \mathrm{~h}$. This may be because both the polymers and the drug used in the formulation are hydrophilic, which couldn't remain intact throughout the release study. Also, formulation F5 with poloxamer 68, could not sustain the release of the drug as the main requirement of floating drug delivery system is to form a cohesive gel barrier, with this polymer could not make [35]. Hence, both these polymers are not the good release-retarding polymers for preparing matrix formulations of the hydrophilic drug. Although, the change in the composition for both the polymers can be tried to get the desired release pattern of the drug. Formulation F6 prepared with only HPMC K15M showed delayed release of the drug, which is contradictory to the earlier findings where it was said that weakly basic drugs gives high release at lower $\mathrm{pH}$ when prepared with HPMC matrix alone $[33,36]$. The formulation prepared with HPMC and sodium alginate showed delayed release. This may be because of less hydration of sodium alginate and also because in acidic $\mathrm{pH}$ it doesn't contribute to the matrix erosion and hence the release of the drug [31]. Pullulan can be used for various coatings of the formulation [37]. In present research an attempt was made to check the ability of pullulan as release regarding polymer for floating formulation, but Formulations F3, with pullulan also showed delayed release of the drug which was almost same as that of formulation F6, this means that the presence of pullulan doesn't have much effect on the 
release pattern of metformin from the polymeric matrix system.

\section{CONCLUSION}

Although, all the combinations tried above can be optimized by changing the amount of significant variables in the formulations. But the batches containing kappa-carrageenan polymer showed better release as compared to all other formulations. Moreover, the swelling index and adhesion retention of this formulation was better than all other formulations, which ensures the retention of the formulation in the stomach. Kappa carrageenan and sodium alginate, both are anionic polymers still the release pattern of the drug found to be different in both of them which indicates that the $\mathrm{pH}$ of the medium changes the properties of the polymer and hence the release of the drug. The basic mechanism of the gastro retention for the formulation is floatation, but in the case of low level of fluid in the stomach, the mechanisms like mucoadhesion and swelling can retain the formulation at the required site, which can be better achieved by formulation with kappa-carrageenan. It has been already reported that the gastro restive formulations prepared by using the carrageenans can modify the properties of polymeric matrices, to obtain tailor-made materials for drug delivery systems. Hence, this combination of polymers can be further evaluated by applying appropriate design, to obtain the optimized gastro retentive formulation of metformin.

\section{Conflicts of Interests}

Declared none

\section{REFERENCES}

[1] Adikwu MU, Yoshikawa Y, Takada K. Pharmacodynamic-pharmacokinetic profiles of metformin hydrochloride from a mucoadhesive formulation of a polysaccharide with antidiabetic property in streptozotocin-induced diabetic rat models. Biomaterials., 2004; 25: 3041-8.

[2] Dunn CJ, Peters DH. Metformin: a review of its pharmacological properties and therapeutic use in noninsulin-dependent diabetes mellitus. Drugs., 1995; 49: 721-49.

[3] Basak SC, Rahman J, Ramalingam M. Design and in vitro testing of a floatable gastro retentive tablet of metformin hydrochloride. Die Pharmazie Int J Pharm Sci., 2007; 62: 145-8.

[4] Porta V, Schramm SG, Kano EK, Koono EE, Armando YP, et al. HPLC-UV determination of metformin in human plasma for application in pharmacokinetics and 
bioequivalence studies. J Pharm

Biomed Anal., 2008; 46: 143-7.

[5] Corti G, Cirri M, Maestrelli F, Mennini N, Mura P. Sustained release matrix tablets of metformin hydrochloride in combination with triacetyl- $\beta$-cyclodextrin. Eur J Pharm Biopharm., 2008; 68: 303-9.

[6] Yadav A, Jain DK. Gastroretentive micro balloons of metformin: Formulation development and characterization. J Adv Pharm Tech Res., 2011; 2: 51-5.

[7] Stepensky D, Friedman M, Srour W, Raz I, Hoffman A. Preclinical evaluation of pharmacokineticpharmacodynamic rationale for oral CR metformin formulation. J Controlled Release., 2001; 7: 107-15.

[8] Colo GD, Falchi S, Zambito Y. In vitro evaluation of a system for $\mathrm{pH}$ controlled peroral delivery of metformin. J Controlled Release., 2002; 80: 119-28.

[9] Ali J, Arora S, Ahuja A, Babbar AK, Sharma RK. Formulation and development of a hydrodynamically balanced system for metformin: in vitro and in vivo evaluation. Eur $\mathbf{J}$ Pharm Biopharm., 2007; 67: 196-201.
[10] Tack-Oon Oh, Ju-Young Kim, JungMyung Ha, Sang-Cheol Chi, YunSeok Rhee. Preparation and in vivo evaluation of highly porous gastro retentive metformin tablets using a sublimation method. Eur J Pharm Biopharm., 2013; 83 Suppl 3:460-7.

[11] Anita KL, Emma L, McConnell LJ, Richard E, Christian S. Decoupling the role of image size and calorie intake on gastric retention of swelling-based gastric retentive formulations: pre-screening in dog model. Int J Pharm., 2012; 431: 90100.

[12] Li H, Hardy RJ, Gu X. Effect of drug solubility on polymer hydration and drug dissolution from polyethylene oxide (PEO) matrix tablets. AAPS PharmSciTech., 2008; 9: 437-43.

[13] Colombo P, Bettini R, Massimo G, Catellani PL, Santi P. Drug diffusion front movement is important in drug release control from swellable matrix tablets. J Pharm Sci., 1995; 84: 991-7.

[14] Pillay V, Fassihi R. A novel approach for constant rate delivery of highly soluble bioactives from a simple monolithic system. J 
Controlled Release., 2000; 67: 6778.

[15] Bettini R, Catellani PL, Santi P, Massimo G, Peppas NA. Translocation of drug particles in HPMC matrix gel layer: effect of drug solubility and influence on release rate. J Controlled Release., 2001; 70: 383-91.

[16] Dorozynski P, Kulinowski P, Mendyk A, Jachowicz R. Gastroretentive drug delivery systems with 1-dopa based on carrageenans and hydroxypropylmethylcellulose. Int J Pharm., 2011; 404: 169-75.

[17] Kshirsagar RV, Jain V, Wattamwar S. Effect of different viscocity grade HPMC polymers on gastro retentive drug delivery of metformin HCL. Int J Appl Pharm., 2009; 1 Suppl 1:44-50.

[18] Mandal U, Pal TK. Formulation and in vitro studies of a fixed-dose combination of a bilayer matrix tablet containing metformin $\mathrm{HCl}$ as sustained release and glipizide as an immediate release. Drug Dev Ind Pharm., 2008; 34: 305-13.

[19] Rawlins EA. Bentley's textbook of pharmaceutics. London, England:
Cassell and Collier Macmillan., 1977.

[20] Defang O, Shufang N, Wei L. In vitro and in vivo evaluation of two extended Release preparations of combination metformin and glipizide. Drug Dev Ind Pharm., 2005; 31: 677-85.

[21] The Indian Pharmacopoeia, Indian Pharmacopoeia Commission, Ministry of Health and family welfare, Government of India., 2007. p. 193.

[22] Dorozynski P, Jachowicz R, Kuligowski P, Kwiecinski S, Szybinski K. The polymers for the preparation of hydrodynamically balanced systems-methods of evaluation. Drug Dev Ind Pharm 2004; 30 Suppl., 9: 947-57.

[23] Patel A, Modasiya M, Shah D, Patel $\mathrm{V}$. Development and in vivo floating behaviour of verapamil $\mathrm{HCl}$ intragastric floating tablets. AAPS PharmSciTech., 2009; 10 Suppl 1: $310-5$.

[24] Rosa M, Zia H, Rhodes T. Dosing and testing in vitro of a bioadhesive and floating drug delivery system for oral application. Int $\mathrm{J}$ Pharm., 1994; 105: 65-70. 
[25] Nakamura F, Ohta R, Machida Y, Nagai T. In vitro and in vivo nasal mucoadhesion of some watersoluble polymers. Int J Pharm., 1996; 134: 173-81.

[26] Perioli L, Ambrogi V, Paganoa C, Scuota S, Rossi C. FG90 chitosan as a new polymer for metronidazole mucoadhesive tablets for vaginal administration. Int J Pharm., 2009; 377: 120-7.

[27] Yuksel N, Kanik AE, Baykara T. Comparison of in vitro dissolution profiles by ANOVA-based, modeldependent and-independent methods. Int J Pharm., 2000; 209: 57-67.

[28] Costa P, Jose MSL. Modeling and comparison of dissolution profiles. Eur J Pharm Sci., 2001; 13: 123-33.

[29] Rao MRP, Sonar GS, Mandsaurwale RR, Vanshiv SD. Evaluation of effervescent floating matrix tablet formulations of salbutamol sulfate using full factorial design. Asian $\mathrm{J}$ Pharm., 2009; 3: 43-9.

[30] Puthori H, Murthy T, Kaushik A, Murthy K. Formulation and evaluation of floating tablets of niacin for sustained release. Asian $\mathrm{J}$ Pharm., 2012., 6 Suppl 1:31-7.
[31] Timmins P, Delargy AM, Howard JR. Optimization and characterization of a $\mathrm{pH}$ independent extended-release hydrophilic matrix tablet. Pharm Dev Technol., 1997; 2 Suppl 1: 25-31.

[32] Campo VL, Kawano DF, da Silva, Jr DB, Carvalho I. Carrageenans: biological properties, chemical modifications, and structural analysis a review. Carbo. Hydr. Polym., 2009; 77: 167-80.

[33] Tatavarti AS, Mehta KA, Augsburger LL, Hoag SW. Influence of methacrylic and acrylic acid polymers on the release performance of weakly basic drugs from sustained release hydrophilic matrices. J Pharm Sci., 2004; 93 Suppl 9: 2319-31.

[34] Singh A, Sharma PK, Malviya R. Release behavior of drugs from various natural gums and polymers. Polim Med., 2011., 41 Suppl 4:7380.

[35] Nayak A, Maji R, Das B. Gastroretentive drug delivery systems: a review. Asian J Pharm Clin Res., 2010, 3: 1-10.

[36] Tatavarti AS, Hoag SW. Microenvironmental $\mathrm{pH}$ modulation based 
release enhancement of a weakly basic drug from hydrophilic matrices. J Pharm Sci., 2006;95 Suppl 7:1459-68.

[37] Izutsu Y, Sogo K, Okamoto S, Tanaka T. Pullulan and sugar coated pharmaceutical composition. US Patent; 1987. 